\title{
Role of Production and Degradation of Serotonin During Development
}

\author{
Kazuhiro Nakamura*,1 $^{*}$ and Hiroyuki Hasegawa ${ }^{2}$ \\ ${ }^{1}$ Department of Pathology, Juntendo University School of Medicine, Tokyo 113-8421, Japan \\ ${ }^{2}$ Department of Biosciences, Teikyo University of Science and Technology, Uenohara, Yamanashi 409-0193, Japan
}

\begin{abstract}
Serotonin (5-hydroxytryptamine [5-HT]) is one of the major neurotransmitter in the brain. Since 5-HT is carried by 5-HT transporters and the biological activities of 5-HT are exerted through 5-HT receptors, disturbed regulation of 5-HT transporters and receptors in the adult brain has been implicated in pathological conditions in central nervous systems. On the other hand, proper 5-HT neurotransmission during development underlies the mature functional architecture of serotonergic neurons, and an increasing body of evidence suggests the involvement of developmental brain disturbances in psychiatric disorders. Genetic mouse models have shown that 5-HT receptors and the 5-HT transporter acting during developmental stages modulate developmental processes. In addition, recent works demonstrated that appropriate 5-HT production and 5-HT degradation during develoment are needed for the development of 5-HT neurons and brain functions in the adult. In this review article, we focus on the importance of activities of tryptophan hydroxylase (TPH), the rate-limiting enzyme in 5-HT biosynthesis and monoamine oxidase A (MAOA), a catabolic enzyme responsible for degradation of 5-HT during development for the brain functions in the adult.
\end{abstract}

Keywords: Serotonin, sensorimotor gating, tryptophan hydroxylase, monoamine oxidase.

\section{INTRODUCTION}

Serotonin (5-hydroxytryptamine [5-HT]) is a crucial neurotransmitter implicated in a variety of physiological and pathological functions in the central nervous systems [1,2]. Numerous studies have suggested associations between various neuropsychiatric disorders and genes that modulate 5HT neurotransmission such as the 5-HT transporter and 5HT receptors [2, 3]. These findings indicate that transport of 5-HT into the cells and signal transduction through 5-HT receptors play central roles in 5-HT-related pathology. Indeed, selective serotonin reuptake inhibitors are used for the treatment for several psychiatric disorders $[2,4]$.

The early expression of neurotransmitters and receptors in the developing brain has brought attention to their potential contribution in modulating neuronal developmental processes. Monoamines are among the first neurotransmitter systems to develop during embryogenesis. In addition to its action as a crucial neurotransmitter in various regions in the adult brain, 5-HT is essential for development of 5-HT neurons in an autocrine manner [5]. Therefore, reduced brain 5HT level during development could disturb the development of 5-HT neurons that underlies adult brain functions. Indeed, genetic mouse models showed the observations that deletion of 5-HT receptors $(5-\mathrm{HT} 1 \mathrm{~A}, 5-\mathrm{HT} 1 \mathrm{~B}, 5-\mathrm{HT} 2 \mathrm{C})$ or the 5-HT transporter cause both developmental defects in the nervous system and behavioral phenotypes in the adult [5].

In addition to such direct "effector" molecules that dictate transport or signal transduction of 5-HT, molecules responsible for 5-HT production or 5-HT degradation are also suggested to be involved in the development of neurons and

*Address correspondence to this author at the Department of Pathology, Juntendo University School of Medicine, Tokyo 113-8421, Japan;

E-mail: kaz@juntendo.ac.jp brain functions in the adult. As the pathway of 5-HT biosynthesis, tryptophan hydroxylase (TPH), a member of a family of pterin-dependent aromatic amino acid hydroxylases, catalyzes the formation of 5-hydroxy- $L$-tryptophan (5-HTP) from $L$-tryptophan, the first step in the biosynthesis of the neurotransmitter 5-HT [6-9]. There are two TPH isoforms. $\mathrm{TPH} 2$ and TPH1 are preferentially expressed in the brain and periphery including the skin, respectively $[10,11]$. Aromatic $L$-amino-acid decarboxylase (AADC) subsequently mediates the production of 5-HT (Fig. 1). A minimal kinetic mechanism for a hydroxylase would involve substrate binding, formation of the hydroxylating intermediate, hydroxylation, and product dissociation. TPH activity is also dependent on the co-substrate. TPH is a monooxygenase, incorporating one atom of oxygen from molecular oxygen into the substrate and reducing the other atom to water. The two electrons required for the reduction of the second atom to water are supplied by the $6 \mathrm{R}$-L-erythro-5,6,7,8-tetrahydrobiopterin (BH4). BH4 acts as co-substrate rather than tightly bound cofactor. Thus, TPH, AADC and BH4 are key factors for the production of 5-HT. Then, the resultant 5-HT needs to be eliminated by degradation. Monoamine oxidases (MAOA and MAOB), the enzymes that de-aminate biogenic amines, are responsible for this step.

In this review article, we will first introduce molecules involved in development of serotonergic system. Then, we will show the defects of neural circuit during development and altered brain functions in the adult induced by inactivation of each serotonergic molecule. Since there are some articles suggesting the importance of 5-HT receptors and the 5-HT transporter during development [5, 12], we particularly focus on the importance of TPH, the rate-limiting enzyme in 5-HT biosynthesis and MAOA, a catabolic enzyme responsible for degradation of 5-HT during development for brain functions in the adult. 


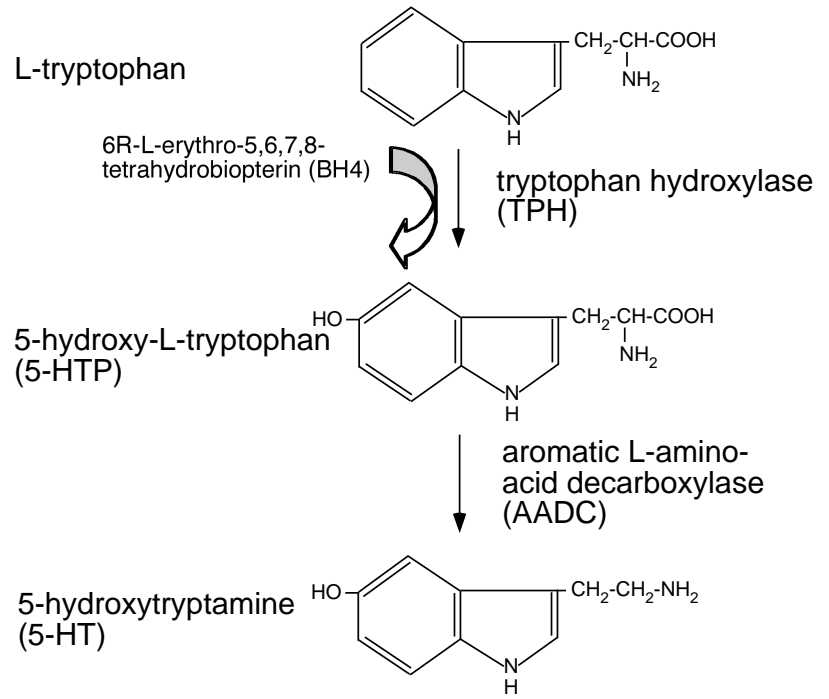

Fig. (1). The pathway of 5-HT biosynthesis.

\section{Development of 5-HT Neurons}

Serotonergic neurons are generated on embryonic days 10 (E10) to E12 in the mouse [13]. One day after their generation, raphe neurons can synthesize 5-HT and begin to extend axon tracts. The full maturation of the axon terminal network is achieved only after birth in rodents [14].

Then, the region of the neural tube in which the 5-HT precursor neurons will be produced is specified. Shh, Fgf4 and Fgf8, which are produced by the notochord, the primitive streak and the mid-hindbrain junction, respectively, act together to specify 5-HT precursor cells [15]. Analysis of a constitutively active form of Smoothened, an Shh receptor, transgenic mouse line indicated a role of the Shh receptor in the dorsoventral control of the 5-HT phenotype [16]. In addition, Otx2 may be responsible for the formation of 5-HT neurons $[17,18]$.

Then, recent study by Jacob et al. showed exciting results that how visceral motor neurons (VMNs) and serotonergic neurons are sequentially generated from a common progenitor pool in the vertebrate hindbrain, and found that the forkhead transcription factor Foxa2, acting in progenitors, is essential for the transition from VMN to serotonergic neurogenesis [19]. Foxa2 activates the switch through a temporal cross-repressive interaction with paired-like homeobox $2 \mathrm{~b}$ (Phox 2b), the VMN progenitor determinant. Moreover, the subsequent differentiation of central serotonergic neurons required both the suppression of VMN neurogenesis and the induction of downstream intrinsic determinants of serotonergic identity by Foxa2.

Next stage is the establishment of the enzymatic machinery that is necessary for the production and metabolism of 5-HT. Proper development and maintenance of the 5-HT transmitter system requires expression of numerous proteins that together define the mature phenotypes of 5-HT neurons. 5-HT biosynthesis by TPH and reuptake of the transmitter by the 5-HT transporter constitute two essential functions of 5-HT neurons that must be coupled for proper serotonergic synaptic transmission. Notably, expression of the TPH and the 5-HT transporter genes is restricted nearly exclusively to 5-HT neurons [20-22]. The more broadly expressed AADC and vesicular monamine transporter 2 (VMAT2) are required for the second and final step of 5-HT synthesis and for packaging of 5-HT in synaptic vesicles, respectively. The expression of a transcription factor Pet1 is strictly limited to the raphe nuclei, and appears one day before the serotonergic neurons can be identified. Pet1 could be required for the terminal differentiation of most of the raphe neurons by activating the transcription of the genes responsible for 5-HT production (TPH and AADC) and 5-HT transport (the 5-HT transporter and VMAT) [23, 24]. Indeed, counts of 5-HT immunoreactive cell bodies indicated an $80 \%$ deficiency in the rostral and caudal domains of Pet-1 null mice, which was caused by failure to differentiate into mature serotonergic neurons in the raphe nuclei. The remaining neurons show reduced expression of TPH and the 5-HT transporter in Pet1-deficient mice [25].

\section{Involvement of 5-HT Production and 5-HT Degrada- tion During Development in Morphological Changes and Animal Behavior}

The findings seen in a transcription factor Pet1 null mice provide a supporting evidence that disorganization of serotonergic system during development leads to morphological or functional changes of 5-HT neurons, thereby resulting in behavioral alterations in the adult. Pet1 activates the transcription of the genes responsible for 5-HT production (TPH and AADC) and 5-HT transport (the 5-HT transporter and VMAT). Pet-1 null mice have generally normal health, activity, motor coordination and learning. However, the standard resident-intruder assay of isolation-induced intermale aggression revealed increased aggression in male Pet-1 null mice. The elevated plus maze test also revealed anxietylike behavior in adults [25]. The authors suggested that the increased anxiety-like behavior of Pet-1 null mice may result at least in part from a deficiency of serotonergic innervation of forebrain.

\subsection{5-HT Production}

Among TPH, AADC and $\mathrm{BH} 4$, the effects of inactivation of TPH on neuronal architecture and behavior are well studied. Injection of drugs that deplete 5-HT during embryonic stages resulted in several kinds of defects in neural development. Involvement of 5-HT in neurogenesis was suggested long time ago by Lauder and Krebs. They tested by administering a TPH blocker, $p$-chlorophenylalanine (PCPA) to pregnant rats and dating the time of last cell division for fetal neurons using long survival $3 \mathrm{H}$-thymidine autoradiography. PCPA specifically retarded the onset of neuronal differentiation in brain regions known to contain 5-HT terminals or to have a high 5-HT content in the adult (5-HT target cells) [26].

Since the cerebral cortex is widely innervated by 5 -HTcontaining axons originating from neurons in the raphe nuclei, appropriate amount of 5-HT during development likely underlie the development of cortical neurons and dendritic arborization [27, 28]. Vitalis et al. analyzed the short- and long-term consequences of embryonic 5-HT depletion on the development of the cerebral neocortex of the rat using PCPA during the E12-17 stage of embryonic development, when major events in corticogenesis take place [29]. They found that E12-17 PCPA treatment altered the maturation of py- 
ramidal neurons of layers III and V of the somatosensory cortex, with these cells displaying reduced dendritic arborization and complexity. They also showed that PCPA treatment transiently altered the incorporation in the cortical plate of interneurons derived from the caudal ganglionic eminence, and persistently affected the differentiation of a subpopulation expressing calretinin and/or cholecystokinin. This study suggests the crucial role of 5-HT during embryonic stages in the development of cortical neurons.

The importance of 5-HT after birth for cortical interneuron development was also observed. Depletion of neocortical serotonin afferents with monoamine neurotoxin 5,7dihydroxytryptamine on the date of birth \{postnatal day 0 (P0) \} resulted in a permanent alteration of the dendritic arborization of calretinin-containing interneurons, and a transient delay of parvalbumin and calbindin expression in a number of cortical neurones during the second postnatal week [30]. The involvement of 5-HT in the development of cortical neurons was also assessed using ectopic transplants of E14 primordial neocortex from rats. Petrova and Otellin suggested that 5-HT might promote survival of the transplanted neuroepithelial cells and their differentiation into nerve cells, and might be involved in the regulation of their proliferation [31].

The disturbed 5-HT production during development affects not only neural organization but also brain function in the adult. A recent study demonstrated that TPH is implicated in the mature functional architecture for sensorimotor gating via appropriate actin polymerization [32, 33]. Sensorimotor gating is a brain function, by which excess or trivial stimuli are screened or "gated out" of awareness by central inhibitory mechanisms in the early stage of information processing [34]. Sensorimotor gating is theoretically and reliably measured by the degree of prepulse inhibition (PPI), the suppression of the startle response by a relatively weak preceded stimulus [34, 35]. Reduced PPI is shared by several common psychiatric and neurodegenerative disorders such as schizophrenia, Huntington's disease, Tourette's syndrome, schizotypal disorder, obsessive-compulsive disorder, and attention-deficit hyperactivity disorder [36]. Based on the theory, gating deficits could lead to a breakdown of cognitive integrity and difficulty in distinguishing self from nonself $[37,38]$ and could reflect the "flooding" by sensory overload and cognitive fragmentation seen in schizophrenia [39]. Indeed, antipsychotic agents used in the treatment of schizophrenia can increase PPI and reverse drug-induced deficits in animal models of sensorimotor gating [40]. Furthermore, some studies have shown that deficits in sensorimotor gating correlate with negative symptoms and thought disorder in schizophrenic patients [41-43]. Agonists and antagonists of 5-HT receptors such as $5-\mathrm{HT}_{1 \mathrm{~A}}$ agonist $8-\mathrm{OH}-$ DPAT, 5- $\mathrm{HT}_{1 \mathrm{~A} / 1 \mathrm{~B}}$ agonist RU24969, selective $5-\mathrm{HT}_{1 \mathrm{~A}}$ antagonist WAY100,635 and 5- $\mathrm{HT}_{2}$ agonist DOI modulate PPI in the adult [44], and a progressive increase in PPI occurs by 8 years of age [45-47]. Therefore, it is likely that the formation of mature neural circuits for sensorimotor gating is achieved during childhood, and changes in brain 5-HT content during development could disturb the development of 5HT neurons involved in the PPI circuit, which might impair adult PPI.

Pharmacological experiments defined the sensitive period for the development of PPI (Fig. 2) [32, 33]. The blockade of TPH using PCPA from P21 to P24, but not earlier or later time points, perturbed the PPI of New Zealand Black (NZB)
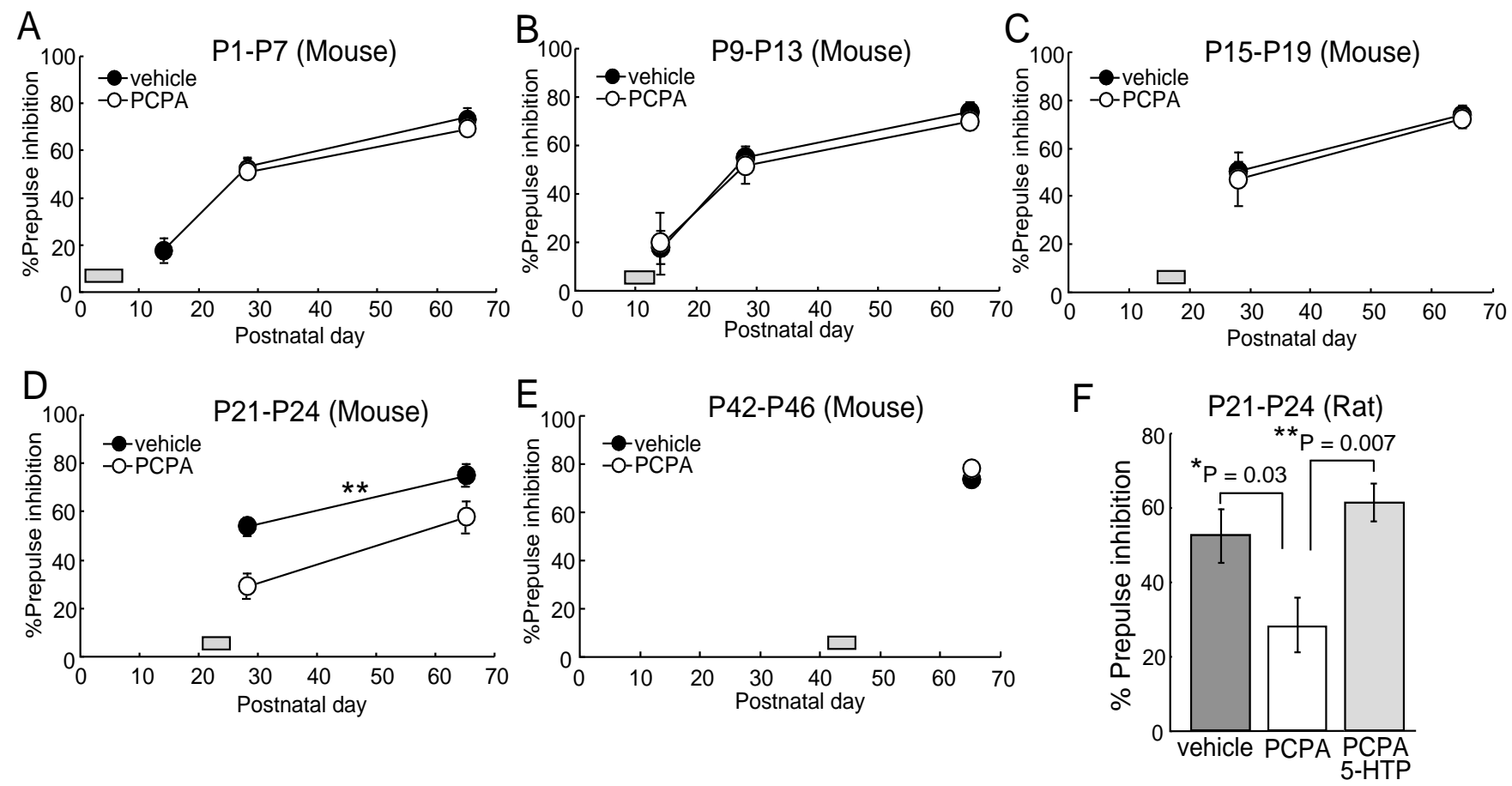

Fig. (2). The sensitive period when 5-HT is required for the maturation of sensorimotor gating is from P21 to P24 in rodents. A to E- Vehicle or PCPA was given to mice during P1-P7 (A), P9-P13 (B), P15-P19 (C), P21-P24 (D) or P42-P46 (E), and the percentage of PPI at a prepulse intensity of 80-dB was measured at P14, P28 and/or P65. Closed squares indicate the period drugs were given. F- Percentage of prepulse inhibition at a prepulse intensity of $65-\mathrm{dB}$ in adult rats administered vehicle, PCPA or PCPA plus 5-HTP from P21 to P24. Error bars represent the mean \pm SEM. $* \mathrm{P}<0.05$, **P $<0.01$, (J Mol Biol 2006; 363: 345-54. Copyright by Elsevier). 
mice with normal PPI levels. Reduced PPI in adulthood was also observed when we injected PCPA during P21-P24 into rats. When the 5-HT precursor 5-HTP was given together with PCPA from P21 to P24, the attenuation of PPI was rescued in these rats. Therefore, the sensitive period when proper 5-HT levels are required for PPI in adulthood proved to be from P21 to P24 in rodents.

\subsection{5-HT Degradation (MAOA)}

A line of transgenic mice in which transgene integration caused a deletion in the gene encoding MAOA, a catabolic enzyme responsible for degradation of 5-HT, was used for an animal model of MAOA deficiency. These MAOA-deficient mice caused 5-HT accumulation (up to ninefold) during early postnatal period and cytoarchitectural changes in the somatosensory cortex [48]. Behavioral studies revealed pup behavioral alterations, including trembling, difficulty in righting, and fearfulness. These behavioral changes were reasonably reversed by the 5-HT synthesis inhibitor PCPA. Adults manifested a distinct behavioral syndrome, including enhanced aggression in males [48].

The animal model of MAOA deficiency provided a good opportunity to test the effect of alterations of 5-HT levels during development on brain wiring and the behavioral changes in the adult. A complete absence of barrels in the somatosensory cortex seen in MAOA-deficient mice is also caused by pharmacological inhibition of MAOA in wild type mice. The MAOA inhibitor clorgyline was administered to mice of the outbred strain OF1 for various time periods between E15 and P7, and the barrel fields were analyzed in P10 and adult mice. Clorgyline treatments from E15 to P7 or from P0 to P7 disrupted the formation of barrels in the anterior snout representation and in parts of the posteromedial barrel subfield. Interestingly, clorgyline treatments only during embryonic life or starting on P4 caused no detectable abnormalities. These observations indicate that the critical period for abnormal brain wiring in the somatosensory cortex proved first postnatal week, and the inhibition of MAOA during embryonic days or in the adult does not result in visible effects [48, 49]. For the effect of normalization of 5-HT levels on the behavior in the mutant mice, treatment with the inhibitor of 5-HT synthesis PCPA from birth to P14 in MAOA-deficient mice greatly improved their walking posture on the beam. Therefore, the behavioral change seen in beam walking of MAOA-deficient mice were rescued when 5-HT levels during early postnatal period were controlled [50]. Although MAOA also oxidizes norepinephrine, future works will further strengthen the specific relationship among the behavioral changes, MAOA and 5-HT levels.

\section{CONCLUSION}

The organization of proper neural circuits responsible for each brain function is sensitive to neuronal activities during the well-defined interval during development. 5-HT is essential for the development of 5-HT neurons in an autocrine manner. Further precise studies will clarify whether the anatomical defects led by reduced 5-HT production during development are the fine-tuning of 5-HT neuronal connections and are distinct from those seen in 5-HT receptors or the 5HT transporter. The functional relationship between the subtle changes in 5-HT neuronal networks in the adult induced by reduced 5-HT production during development and disor- ganized brain functions in the adult may be also investigated. In addition, elaborate experimental designs will define the sensitive period for the activities of molecules responsive for 5-HT production and 5-HT degradation during development that are required for organization of several other brain functions in the adult. Such information might serve as substitution therapy of serotonergic substances during development to prevent prospective disorganized brain functions in the adult, providing the association between the polymorphisms in the molecule dictating 5-HT production or 5-HT degradation and the defect of brain functions can be confirmed with careful examinations.
ABBREVIATIONS

AADC $=$ Aromatic $L$-amino-acid decarboxylase
BH4 $=6$ - $L$-erythro-5,6,7,8-tetrahydrobiopterin
5 -HT $=5$-hydroxytryptamine
5-HTP $=5$-hydroxy- $L$-tryptophan
MAOA $=$ monoamine oxidase A
PCPA $=p$-chlorophenylalanine
Phox2b $=$ paired-like homeobox $2 \mathrm{~b}$
PPI $=$ prepulse inhibition
TPH $=$ tryptophan hydroxylase
VMAT $=$ vesicular monamine transporter
VMN $=$ visceral motor neurons

\section{REFERENCES}

[1] Lesch KP. Gene-environment interaction and the genetics of depression. J Psychiatry Neurosci 2004; 29: 174-84.

[2] Lucki I. The spectrum of behaviors influenced by serotonin. Biol Psychiatry 1998; 44: 151-62.

[3] Lesch KP, Bengel D, Heils A, et al. Association of anxiety-related traits with a polymorphism in the serotonin transporter gene regulatory region. Science 1996; 274: 1527-31.

[4] Gainetdinov RR, Caron MG. Monoamine transporters: from genes to behavior. Annu Rev Pharmacol Toxicol 2003; 43: 261-84.

[5] Gaspar P, Cases O, Maroteaux L. The developmental role of serotonin: news from mouse molecular genetics. Nat Rev Neurosci 2003; 4: 1002-12.

[6] Grahame-Smith DG. The biosynthesis of 5-hydroxytryptamine in brain. Biochem J 1967; 105: 351-60.

[7] Hosoda S, Glick D. Biosynthesis of 5-hydroxytryptophan and 5hydroxytryptamine from tryptophan by neoplastic mouse mast cells. Biochim Biophys Acta 1965; 111: 67-78.

[8] Ichiyama A, Nakamura S, Nishizuka Y, Hayaishi O. Enzymic studies on the biosynthesis of serotonin in mammalian brain. J Biol Chem 1970; 245: 1699-709.

[9] Lovenberg W, Jequier E, Sjoerdsma A. Tryptophan hydroxylation: measurement in pineal gland, brainstem, and carcinoid tumor. Science $1967 ; 155$ : 217-9.

[10] Slominski A, Wortsman J, Tobin DJ. The cutaneous serotoninergic/melatoninergic system: securing a place under the sun. FASEB J 2005; 19: 176-94.

[11] Walther DJ, Peter JU, Bashammakh S, et al. Synthesis of serotonin by a second tryptophan hydroxylase isoform. Science 2003; 299: 76.

[12] Gross C, Zhuang X, Stark K, et al. Serotonin1A receptor acts during development to establish normal anxiety-like behaviour in the adult. Nature 2002; 416: 396-400.

[13] Levitt P, Rakic P. The time of genesis, embryonic origin and differentiation of the brain stem monoamine neurons in the rhesus monkey. Brain Res 1982; 256: 35-57.

[14] Lidov HG, Molliver ME. An immunohistochemical study of serotonin neuron development in the rat: ascending pathways and terminal fields. Brain Res Bull 1982; 8: 389-430. 
[15] Brodski C, Weisenhorn DM, Signore M, et al. Location and size of dopaminergic and serotonergic cell populations are controlled by the position of the midbrain-hindbrain organizer. J Neurosci 2003; 23: 4199-207.

[16] Hynes M, Ye W, Wang K, et al. The seven-transmembrane receptor smoothened cell-autonomously induces multiple ventral cell types. Nat Neurosci 2000; 3: 41-6.

[17] Puelles E, Annino A, Tuorto F, et al. Otx2 regulates the extent, identity and fate of neuronal progenitor domains in the ventral midbrain. Development 2004; 131: 2037-48.

[18] Vernay B, Koch M, Vaccarino F, et al. Otx2 regulates subtype specification and neurogenesis in the midbrain. J Neurosci 2005; 25: 4856-67.

[19] Jacob J, Ferri AL, Milton C, et al. Transcriptional repression coordinates the temporal switch from motor to serotonergic neurogenesis. Nat Neurosci 2007; 10: 1433-9.

[20] Hansson SR, Mezey E, Hoffman BJ. Serotonin transporter messenger RNA in the developing rat brain: early expression in serotonergic neurons and transient expression in non-serotonergic neurons. Neuroscience 1998; 83: 1185-201.

[21] Rattray M, Michael GJ, Lee J, Wotherspoon G, Bendotti C, Priestley JV. Intraregional variation in expression of serotonin transporter messenger RNA by 5-hydroxytryptamine neurons. Neuroscience 1999; 88: 169-83.

[22] Rind HB, Russo AF, Whittemore SR. Developmental regulation of tryptophan hydroxylase messenger RNA expression and enzyme activity in the raphe and its target fields. Neuroscience 2000; 101: 665-77.

[23] Hendricks T, Francis N, Fyodorov D, Deneris ES. The ETS domain factor Pet-1 is an early and precise marker of central serotonin neurons and interacts with a conserved element in serotonergic genes. J Neurosci 1999; 19: 10348-56.

[24] Pfaar H, von Holst A, Vogt Weisenhorn DM, Brodski C, Guimera $\mathrm{J}$, Wurst W. mPet-1, a mouse ETS-domain transcription factor, is expressed in central serotonergic neurons. Dev Genes Evol 2002; 212: 43-6.

[25] Hendricks TJ, Fyodorov DV, Wegman LJ, et al. Pet-1 ETS gene plays a critical role in 5-HT neuron development and is required for normal anxiety-like and aggressive behavior. Neuron 2003; 37: 233-47.

[26] Lauder JM, Krebs H. Serotonin as a differentiation signal in early neurogenesis. Dev Neurosci 1978; 1: 15-30.

[27] Levitt P, Harvey JA, Friedman E, Simansky K, Murphy EH. New evidence for neurotransmitter influences on brain development. Trends Neurosci 1997; 20: 269-74.

[28] Vitalis T, Parnavelas JG. The role of serotonin in early cortical development. Dev Neurosci 2003; 25: 245-56.

[29] Vitalis T, Cases O, Passemard S, Callebert J, Parnavelas JG. Embryonic depletion of serotonin affects cortical development. Eur J Neurosci 2007; 26: 331-44.

[30] Durig J, Hornung JP. Neonatal serotonin depletion affects developing and mature mouse cortical neurons. Neuroreport 2000; 11: 8337.

[31] Petrova ES, Otellin VA. Serotonin is involved in the regulation of histogenetic processes in rat embryonic neocortex. Bull Exp Biol Med 2007; 143: 372-5.
[32] Nakamura K, Koyama Y, Takahashi K, et al. Requirement of tryptophan hydroxylase during development for maturation of sensorimotor gating. J Mol Biol 2006; 363: 345-54.

[33] Nakamura K, Sugawara Y, Sawabe K, et al. Late developmental stage-specific role of tryptophan hydroxylase 1 in brain serotonin levels. J Neurosci 2006; 26: 530-4.

[34] Braff DL, Geyer MA. Sensorimotor gating and schizophrenia. Human and animal model studies. Arch Gen Psychiatry 1990; 47 181-8.

[35] Graham FK. Presidential Address, 1974. The more or less startling effects of weak prestimulation. Psychophysiology 1975; 12: 23848 .

[36] Braff DL, Geyer MA, Swerdlow NR. Human studies of prepulse inhibition of startle: normal subjects, patient groups, and pharmacological studies. Psychopharmacology (Berl) 2001; 156: 234-58.

[37] Karper LP, Freeman GK, Grillon C, Morgan CA, 3rd, Charney DS, Krystal JH. Preliminary evidence of an association between sensorimotor gating and distractibility in psychosis. J Neuropsychiatry Clin Neurosci 1996; 8: 60-6.

[38] Vollenweider FX, Geyer MA. A systems model of altered consciousness: integrating natural and drug-induced psychoses. Brain Res Bull 2001; 56: 495-507.

[39] Braff DL. Information processing and attention dysfunctions in schizophrenia. Schizophr Bull 1993; 19: 233-59.

[40] Swerdlow NR, Geyer MA. Clozapine and haloperidol in an animal model of sensorimotor gating deficits in schizophrenia. Pharmacol Biochem Behav 1993; 44: 741-4.

[41] Braff DL, Swerdlow NR, Geyer MA. Symptom correlates of prepulse inhibition deficits in male schizophrenic patients. Am J Psychiatry 1999; 156: 596-602.

[42] Perry W, Braff DL. Information-processing deficits and thought disorder in schizophrenia. Am J Psychiatry 1994; 151: 363-7.

[43] Perry W, Geyer MA, Braff DL. Sensorimotor gating and thought disturbance measured in close temporal proximity in schizophrenic patients. Arch Gen Psychiatry 1999; 56: 277-81.

[44] Geyer MA, Krebs-Thomson K, Braff DL, Swerdlow NR. Pharmacological studies of prepulse inhibition models of sensorimotor gating deficits in schizophrenia: a decade in review. Psychopharmacology (Berl) 2001; 156: 117-54.

[45] Anthony BJ, Graham FK. Evidence for sensory-selective set in young infants. Science 1983; 220: 742-4.

[46] Ornitz EM, Guthrie D, Kaplan AR, Lane SJ, Norman RJ. Maturation of startle modulation. Psychophysiology 1986; 23: 624-34.

[47] Ornitz EM, Guthrie D, Sadeghpour M, Sugiyama T. Maturation of prestimulation-induced startle modulation in girls. Psychophysiology $1991 ; 28: 11-20$

[48] Cases O, Seif I, Grimsby J, et al. Aggressive behavior and altered amounts of brain serotonin and norepinephrine in mice lacking MAOA. Science 1995; 268: 1763-6.

[49] Vitalis T, Cases O, Callebert J, et al. Effects of monoamine oxidase A inhibition on barrel formation in the mouse somatosensory cortex: determination of a sensitive developmental period. J Comp Neurol 1998; 393: 169-84

[50] Salichon N, Gaspar P, Upton AL, et al Excessive activation of serotonin (5-HT) 1B receptors disrupts the formation of sensory maps in monoamine oxidase a and 5-ht transporter knock-out mice. J Neurosci 2001; 21: 884-96.

(C) Nakamura and Hasegawa; Licensee Bentham Open

This is an open access article licensed under the terms of the Creative Commons Attribution Non-Commercial License (http://creativecommons.org/licenses/by-nc/3.0/) which permits unrestricted, non-commercial use, distribution and reproduction in any medium, provided the work is properly cited. 